\title{
El comportamiento de la pareja influye en el cambio hacia conductas relacionadas con la salud
}

Partner's behavior influences health behavior change

\section{Objetivos}

Examinar el impacto que tiene el cambio del comportamiento de un cónyuge en relación a conductas (o hábitos de vida) saludables, sobre dicho aspecto en su pareja.

\section{Diseño, lugar y pacientes}

Estudio de cohorte prospectiva realizado en el Reino Unido. Fueron incluidas 3.722 parejas heterosexuales (casadas o convivientes) de la cohorte ELSA (English longitudinal study of ageing) de adultos mayores de 50 años, que presentaran al menos un comportamiento de salud poco saludable (tabaquismo, sedentarismo) o sobrepeso en dos evaluaciones consecutivas. Se realizó un seguimiento prospectivo desde el año 2002 al 2014.

\section{Medición de resultados principales}

El resultado primario de interés consistió en identificar cambios en comportamientos de hábitos saludables entre ambos miembros de la pareja; y el resultado secundario, en identificar el cambio cruzado, es decir, si el cambio positivo en un hábito (ej. tabaquismo) produce cambios positivos del comportamiento en otro hábito relacionado (ej. sobrepeso u obesidad).

\section{Evaluación de factores pronósticos}

El seguimiento se realizó cada dos años para tabaquismo y actividad

Tabla 1. Influencia del comportamiento de la pareja en el cambio del comportamiento vinculado con hábitos de vida saludables.
Jackson SE, y col. JAMA Intern Med. 2015;175(3):385-92. física, mediante entrevistas virtuales y cuestionarios. Para evaluar el sobrepeso se recopilaron datos antropométricos cada cuatro años. El ajuste por posibles confundidores incluyó comorbilidades tales como el diagnóstico de cáncer, diabetes, accidente cerebrovascular, infarto agudo de miocardio y patologías crónicas. Se calculó el odds ratio (OR) para realizar cambios positivos en el comportamiento (ej. pasaje de tabaquismo activo a cesación) con respecto al comportamiento de la pareja entre ambos puntos de la evaluación.

\section{Resultados principales}

De las 5.746 parejas de la cohorte ELSA, fueron incluidas 3.722 parejas con al menos un miembro con un comportamiento poco saludable en primera instancia. Se recolectó información de tabaquismo en 3.555 parejas, de actividad física en 3.520 y de peso en 1.556 . En el caso de la actividad física, para individuos sedentarios, tener una pareja habitualmente activa se asoció a mayor incremento en la actividad física del cónyuge. Sin embargo, tener una pareja que era sedentaria y se volvió activa se relacionó aún con mejores resultados. Con el tabaquismo se observaron cambios similares. Con respecto al sobrepeso, tener una pareja con un índice de masa corporal (IMC) normal no se relacionó con mejores resultados, pero sí el tener una pareja con sobrepeso que bajó de peso durante el seguimiento (ver tabla 1).

\begin{tabular}{c|c|c|c}
\multicolumn{1}{c}{$\begin{array}{c}\text { Conducta } \\
\text { Tabaquismo }\end{array}$} & Comportamiento de la pareja & $\begin{array}{c}\text { Proporción de sujetos que realizaron un cambio a } \\
\text { comportamiento saludable (\%) }\end{array}$ & $\begin{array}{c}\text { OR (IC 95\%) } \\
1 \text { (Referencia) }\end{array}$ \\
\hline \multirow{3}{*}{ Actividad física } & Fumador estable & 7,7 & $3,84(2,09 \mathrm{a} 7,06)$ \\
\cline { 2 - 4 } & No fumador & 23,3 & $11,82(4,84 \mathrm{a} 28,9)$ \\
\cline { 2 - 4 } & Dejó de fumar & 48,4 & 1 \\
\cline { 2 - 4 } & Inactivo & 25,9 & $2,75(1,98$ a 3,81$)$ \\
\cline { 2 - 4 } & Activo habitual & 54 & $5,28(3,7 \mathrm{a} 7,5)$ \\
\cline { 2 - 4 } & Se volvió activo & 67,3 & 1 \\
\cline { 2 - 4 } & Sobrepeso estable & 9,9 & $1,34(0,86$ a 2,08) \\
\cline { 2 - 4 } & Peso normal estable & 12,6 & $3,05(1,96$ a 4,74$)$ \\
\hline
\end{tabular}

\section{Conclusiones}

Si bien tener una pareja que ya adhiere a hábitos de vida saludable está asociado a cambios positivos en el comportamiento de la otra, tener una pareja que realiza cambios positivos en este sentido, está relacionado con aún mejores resultados. No se evidenció que los cambios positivos en un comportamiento generen cambios en otros comportamientos relacionados.

Fuente de financiamiento: US National Institute of Aging.

\section{Comentario}

Si bien hay estudios que sugieren que el hecho de tener una pareja saludable influencia de manera positiva la realización de cambios en el otro miembro, no demuestran que realizar intervenciones en la práctica clínica sobre ambos miembros sea más beneficioso'. Sin dudas el ámbito familiar es de crucial importancia para adquirir hábitos saludables. Con respecto a la obesidad, si bien la Sociedad Argentina de Nutrición realiza recomendaciones basadas en las guías NICE (The National Institute for Health and Care Excellence), que proponen estrategias de índole individual y grupal, se hace hincapié en la importancia de intervenir sobre las familias sobre todo cuando este problema afecta a niños o adolescentes, pero no se menciona específicamente el hecho de realizar intervenciones en ad hoc en las parejas $^{2,3}$. Con respecto al tabaquismo, ocurre algo similar. Las guías del Ministerio de Salud de la Nación Argentina proponen estrategias individuales o grupales pero no hacen mención a la posibilidad de intervenir en los conyugues ${ }^{4}$.

\section{Conclusiones del comentador}

Sin dudas el contexto social de los individuos es importante para el desarrollo de sus hábitos y costumbres. Dado que el cambio positivo en uno de los miembros de la pareja puede generar cambios en el otro miembro, queda como interrogante la posibilidad de implementar intervenciones en ambos miembros de la pareja en lugar de hacerlo en forma individual.

Sofía Gabriela Sciarreta [ Servicio Medicina familiar y Comunitaria del Hospital Italiano de Buenos Aires sofia.sciarreta@hospitalitaliano.org.ar ]

Sciarreta SG. El comportamiento de la pareja influye en el cambio hacia conductas relacionadas con la salud. Evid Act Pract Ambul. $2017 ; 20(1): 14$. Comentado de: Jackson SE, y col. The Influence of Partner's Behavior on Health Behavior Change: The English Longitudinal Study of Ageing. JAMA Intern Med. 2015;175(3):385-92. PMID: 25599511.

\section{Referencias}

1. Barnett I y col. How do couples influence each other's physical activity behaviours in retirement? An exploratory qualitative study. BMC Public Health. 2013;13:1197.

2. National Institute for Health and Care Excellence. Managing overweight and obesity among children and young people: lifestyle weight management services. (Public Health Guideline PH47). 2013. Disponible en: https://www.nice.org.uk/guidance/ph47

3. National Institute for Health and Care Excellence. Physical activity: brief advice for adults in primary care. (Public Health Guideline PH44). 2013. Disponible en: https://www.nice.org.uk/guidance/ph44 4. Ministerio de Salud de la Nación. Guía de Práctica Clínica Nacional de Tratamiento de la Adicción al Tabaco. Buenos Aires, 2014. Disponible en: http://www.msal.gob.ar/images/stories/bes/graficos/0000000536cnt-2014-09_guia-tratamiento-adiccion-tabaco-2014.pdf 